\title{
Long-term functional benefits of selective dorsal rhizotomy for spastic cerebral palsy
}

\author{
Clinical article
}

\author{
Roy W. R. Dudley, M.D., Ph.D., ${ }^{1}$ Michele Parolin, M.D., ${ }^{2}$ Bruno Gagnon, M.D., M.Sc., ${ }^{3}$ \\ Rajeet Saluja, M.D., ${ }^{1}$ Rita YaP, M.Sc. ${ }^{4}$ Kathleen Montpetit, M.Sc., ${ }^{5}$ Joanne Ruck, M.Sc., ${ }^{4}$ \\ Chantal Poulin, M.D. ${ }^{6}$ Marie-Andrée Cantin, M.D., ${ }^{7}$ Thierry E. Benaroch, M.D., ${ }^{8}$ \\ and Jean-Pierre Farmer, M.D. ${ }^{1}$
}

\begin{abstract}
${ }^{I}$ Division of Neurosurgery, Department of Pediatric Surgery, Montreal Children's Hospital, McGill University, Montreal, Quebec, Canada; ${ }^{2}$ Department of Neurosurgery, Anna Meyer Hospital, Florence, Italy; ${ }^{3}$ Division of Clinical Epidemiology, Royal Victoria Hospital; ${ }^{4}$ Physical Therapy and ${ }^{5}$ Occupational Therapy, Shriners Hospital for Children - Canada; ${ }^{6}$ Department of Neurology, Montreal Children's Hospital; ${ }^{7}$ Division of Orthopedic Surgery, Department of Surgery, Shriners Hospital for Children, Sainte-Justine's Hospital, Université de Montreal; and ${ }^{8}$ Division of Orthopedic Surgery, Department of Pediatric Surgery, Montreal Children's Hospital, McGill University, Montreal, Quebec, Canada
\end{abstract}

Object. Large-scale natural history studies of gross motor development have shown that children with spastic cerebral palsy (CP) plateau during childhood and actually decline through adolescence. Selective dorsal rhizotomy (SDR) is a well-recognized treatment for spastic $\mathrm{CP}$, but little is known about long-term outcomes of this treatment. The purpose of this study was to assess the durability of functional outcomes in a large number of patients through adolescence and into early adulthood using standardized assessment tools.

Methods. The authors analyzed long-term follow-up data in children who had been evaluated by a multidisciplinary team preoperatively and at 1, 5, 10, and 15 years after SDR. These evaluations included quantitative, standardized assessments of lower-limb tone (Ashworth Scale), Gross Motor Function Measure (GMFM), and performance of activities of daily living (ADLs) by the Pediatric Evaluation of Disability Inventory in children who had been stratified by motor severity using the Gross Motor Function Classification System (GMFCS). In addition, group-based trajectory modeling (GBTM) was used to identify any heterogeneity of response to SDR among these treated children, and to find which pretreatment variables might be associated with this heterogeneity. Finally, a chart review of adjunct orthopedic procedures required by these children following SDR was performed.

Results. Of 102 patients who underwent preoperative evaluations, 97, 62, 57, and 14 patients completed postoperative assessments at 1,5,10, and 15 years, respectively. After SDR, through adolescence and into early adulthood, statistically significant durable improvements in lower-limb muscle tone, gross motor function, and performance of ADLs were found. When stratified by the GMFCS, long-lasting improvements for GMFCS Groups I, II, and III were found. The GBTM revealed 4 groups of patients who responded differently to SDR. This group assignment was associated with distribution of spasticity (diplegia was associated with better outcomes than triplegia or quadriplegia) and degree of hip adductor spasticity (Ashworth score $<3$ was associated with better outcomes than a score of 3), but not with age, sex, degree of ankle plantar flexion spasticity, or degree of hamstring spasticity. In a sample of 88 patients who had complete records of orthopedic procedures and botulinum toxin (Botox) injections, $52(59.1 \%)$ underwent SDR alone, 11 (12.5\%) received only Botox injections in addition to SDR, while 25 patients $(28.4 \%)$ needed further lower-extremity orthopedic surgery after SDR.

Conclusions. In the majority of patients, the benefits of SDR are durable through adolescence and into early adulthood. These benefits include improved muscle tone, gross motor function, and performance of ADLs, as well as a decreased need for adjunct orthopedic procedures or Botox injections. The children most likely to display these long-term benefits are those in GMFCS Groups I, II, and III, with spastic diplegia, less hip adductor spasticity, and preoperative GMFM scores greater than 60 . (http://thejns.org/doi/abs/10.3171/2013.4.PEDS12539)

\section{Key Words • cerebral palsy - spasticity • selective dorsal rhizotomy • long-term follow-up • functional neurosurgery}

\footnotetext{
Abbreviations used in this paper: ADLs $=$ activities of daily living; $\mathrm{CP}=$ cerebral palsy; GBTM = group-based trajectory modeling; GMFCS = Gross Motor Function Classification System; GMFM $=$ Gross Motor Function Measure; PEDI $=$ Pediatric Evaluation of Disability Inventory; PILAR = Predictive Index for Long-Term Ambulation after Rhizotomy; SDR = selective dorsal rhizotomy.
}

I $\mathrm{N} C P$, damage to the immature brain is nonprogressive, but the clinical expression of the disorder changes over time with the growth and development of the child. ${ }^{2}$ The benchmark Ontario Motor Growth Study ${ }^{18}$ and the Adolescent Study of Quality of Life, Mobility, and Exercise ${ }^{10}$ described the natural history of gross mo- 
tor development in untreated $\mathrm{CP}$ using a combination of the GMFM $^{19}$ and the GMFCS. ${ }^{17}$ These studies revealed that most children with spastic $\mathrm{CP}$ plateau in motor development between the ages of 6 and $9,{ }^{18}$ and that motor development in more severely affected children (GMFCS Groups III, IV, and V) actually declines through adolescence and into early adulthood..$^{10}$

Selective dorsal rhizotomy is a well-established treatment for carefully selected children with the spastic form of CP. ${ }^{7}$ Two of 3 randomized control trials and a metaanalysis of those trials have revealed the functional benefits of SDR, at least up to 2 years after surgery. ${ }^{12-14,16,20,23}$ However, no study has reported long-term data on large numbers of patients using standardized functional assessment tools; thus it remains unresolved whether SDR impacts the natural history of gross motor development in CP. Using validated assessment tools, we assessed whether functional benefits of SDR are durable through adolescence and into early adulthood. In addition, we used GBTM to look for heterogeneity of response to SDR among our patients. Our hypothesis was that in well-selected children with spastic CP, SDR would provide longterm functional benefits and protect against adolescent declines in motor development, and that heterogeneity would exist in terms of response to SDR, which would be based on preoperative patient characteristics.

\section{Methods}

\section{Data Collection and Study Population}

We examined data on patients who underwent SDR between 1991 and 2001. The data were initially collected prospectively and are stored in the McGill University Rhozotomy Database. Families provided informed consent for outcomes research purposes. The study population consisted of children with spastic CP evaluated preoperatively and postoperatively for as many as 15 years. Patients selected for surgery had a typical perinatal history, a static disease process, and emerging locomotor function. Patients with underlying low tone, dystonia, and multiple prior orthopedic procedures were excluded. Of 105 patients who had SDR between October 1991 and August 2001, 102 had formal preoperative evaluations, and $97,62,57$, and 14 patients had formal postoperative assessments at 1, 5, 10, and 15 years, respectively.

\section{Patient Subgroups}

The GMFCS is the international standard for classifying the severity of CP.17,19 Patients were categorized preoperatively by age-specific gross motor activity as follows: Group I, walks without restrictions, but has limitations in more advanced gross motor skills; Group II, walks without restrictions and without assisted devices (if $<4$ years of age, the child may use a walker and still be considered Group II), but has limitations walking outdoors and in the community; Group III, walks with assistive mobility devices, and has limitations walking outdoors and in the community; Group IV, self-mobility with limitations, children are transported or use power mobility outdoors and in the community; and Group V, self-mobility is severely limited, even with the use of assistive technology. In our practice we have found that nonambulatory patients in Group $\mathrm{V}$ do not obtain functional gains even with the elimination of spasticity with SDR, and thus we do not offer SDR to these patients at our center. However, these patients may be candidates for intrathecal baclofen pumps or tendon release procedures.

\section{Surgical Procedure and Postoperative Care}

The surgical and rehabilitation protocols have been described previously. ${ }^{14}$ Briefly, anesthesia is induced in all patients by a mixture of sufentanil $(0.2-0.5 \mu \mathrm{g} / \mathrm{kg} / \mathrm{hr})$ and propofol (1-10 mg/kg/hr), which is infused along with nitrous oxide. A short-acting nondepolarizing neuromuscular blocking agent, rocuronium, is used only at induction of anesthesia. Lumbosacral dorsal roots from L-1 to S-2 are exposed through a narrow 5-level lumbar laminotomy flap, which allows us to dissect and stimulate every nerve rootlet before determining which ones contribute most to spasticity and need to be sectioned. Because the procedure is performed at the cauda equina and not the conus, a wide laminectomy is unnecessary.

Stimulation of dorsal roots begins after all infusions have reached a steady state. Once threshold and supramaximal responses of the roots are obtained, the latter are divided into rootlets. Both the neurophysiologist and the physical therapist assess the motor responses to electrical stimulation of sensory rootlets. Based on the evoked stimulation responses, rootlets are sectioned using predefined lesioning criteria outlined previously. ${ }^{14}$ The laminotomy flap is then reattached to the surrounding laminae after placement of an epidural catheter for postoperative delivery of a morphine infusion $(4 \mu \mathrm{g} / \mathrm{kg}$ / hr) containing $0.125 \%$ bupivacaine. After surgery, children are allowed to sit in a chair on the fourth postoperative day. The following day, the patients are transferred to the rehabilitation center, where they receive 6 weeks of intensive inpatient rehabilitation (6 hours/day) devoted to muscle reeducation and strengthening. Thereafter, their rehabilitation continues with standard physiotherapy (3 hours/week) involving stretching and strengthening exercises for the lower extremities and occupational therapy for the first year after surgery. Postoperative stability and control of lower limbs is further enhanced by judicious use of orthotic devices.

In long-term follow-up we have previously demonstrated a very low level of significant spinal deformity (scoliosis or lordosis) in these patients, especially if the surgery is performed before the age of $5 .{ }^{8}$ We attribute this lower level of spinal deformity to the fact that we offer this surgery to ambulatory patients predominantly, that the laminotomy is purposefully narrow given that the stimulation and lesioning is performed on rootlets suspended outside the canal, and finally to the 3-point-perlevel fixation of the laminoplasty.

\section{Functional Assessments}

Functional outcome measures were assessed using the Ashworth Scale, ${ }^{1}$ GMFM-88, ${ }^{17}$ and PEDI. ${ }^{9}$ An experienced team of physiotherapists and occupational therapists took measurements using a consistent technique at 
each patient assessment. The children were examined without braces, orthoses, or walking aids. All assessments were videotaped for analysis of the performance and scoring. The tone of the hip adductors, hamstrings, and ankle plantar flexors was assessed using the modified Ashworth Scale with a 5-point grading system based on the NYU Tone Scale. ${ }^{1}$

The GMFM-88 is a well-validated observational instrument specifically developed to measure gross motor function and change over time in children with $\mathrm{CP} .{ }^{17}$ The 88 items of the GMFM are assessed after observation of the child and are scored on a 4-point ordinal scale. The items are weighted equally and grouped into 5 dimensions: A, lying and rolling; $\mathrm{B}$, sitting; $\mathrm{C}$, crawling and kneeling; D, standing; and E, walking, running, and jumping. Scores for each dimension are expressed as a percentage of the maximum score for that dimension. The total score is obtained by averaging the percentage scores across the 5 dimensions.

The $\mathrm{PEDI}^{9}$ is administered in the form of a structured interview with the child's parents in conjunction with clinical observation of the child by the therapist. Particular attention was given to the self-care and mobility domains of the PEDI. The scores for each PEDI domain were summed for a total raw score. Using PEDI conversion tables, raw scores for individual items can be transformed into normative and/or scaled scores. However, conversion tables for normative scores are only available up to 7.5 years of age and therefore could not be used for this long-term analysis. Scaled scores indicate the child's individual performance and increasing levels of function along a 0 to 100 continuum of task difficulty. We present scaled scores for self-care and mobility domains for the entire patient population and as stratified by GMFCS groups.

\section{Adjunct Orthopedic Procedures}

Many children with spastic $\mathrm{CP}$ require orthopedic procedures, such as tendon release or femoral osteotomies for contractures and bone deformities, or Botox injections to decrease spasticity. ${ }^{5-7,21,22}$ We reviewed the charts of all patients to find which of these procedures our patients had undergone.

\section{Statistical Analysis}

Group-based trajectory modeling, a form of latent class analysis for continuous outcomes, describes the course of an outcome over age or time. ${ }^{15}$ In particular, it is used to identify heterogeneity of response to treatment within a treated population, and to divide a population into subgroups of individuals with similar response trajectories. In addition, it is a well-recognized, statistically sound method of addressing missing data in clinical studies (for example, related to attrition or to fewer patients having reached later time points in the study period). Group-based trajectory modeling was modeled using all data points available preoperatively and at $1,2,3,5$, and 10 years postoperatively with a censored normal model. Two strategies were used to analyze the impact of missing data points on trajectories. First, we compared outcome trajectories at 5 years between children who were assessed at both 5 years and
10 years to those with only the 5-year assessment. Second, we performed a sensitivity analysis by replacing missing data points with a score of change from the baseline calculated from available data at the same time point, using the best absolute change, the worse absolute change, and the 10th, 25th, 75th, and 90th percentiles of change from baseline. Finally, we verified results of trajectory analyses from these 6 sensitivity data sets to understand the impact of various scenarios on our results; for example, if all missing data were as low as the worst observed case, as high as the best observed case, or of the same magnitude as the various percentiles of change (10th, 25th, 75th, and 90th) between these two extremes. Because so few patients had 15 -year follow-up evaluations, they were excluded from the trajectory analysis.

Comparisons were made between models allowing for varying numbers of trajectory groups, as well as for the number of parameters used to model time for each group. Model fit was assessed using the Bayesian information criteria and the Akaike information criteria. A posterior probability of membership in each group was provided for each individual, with good fit arising from a high probability of membership into a single group. Associations with trajectory group were examined by adding covariates into the trajectory models to retain the probabilistic features of the model. These covariates included age, sex, distribution of spasticity (spastic diplegia, triplegia, and quadriplegia), degree of adductor spasticity, hamstring spasticity, and plantar flexion spasticity. Chi-square tests and Mantel-Haenszel chi-square tests of trend were performed using assigned groups. Analyses were conducted using SAS version 9.1 for the trajectory analysis using the SAS-based TRAJ (trajectory) procedure developed by Jones and Nagin. ${ }^{11}$ We used the results of the covariate associations found in the GBTM to create a predictive index for long-term outcomes after SDR.

Other than GBTM, all data were reported as mean \pm SEM. For most comparisons, differences between groups were initially tested by 1-way repeated measures ANOVA, with post hoc application of the Holm-Sidak test where appropriate. For Ashworth scale tone data we used an ANOVA on ranks (clinical groupings) with a post hoc Dunn test. Statistical significance was defined as $\mathrm{p} \leq$ 0.05 . On several occasions a child did not participate in all measurements, and/or a particular measurement was not recorded in the database by the evaluator. In these incidences data were missing for that particular test at that particular time point. Therefore, for the statistical analysis, numbers of observations in each group, even within the same test, differed at times. However, each statistical analysis was based on all available data for the particular parameter in question. The statistical analysis program SigmaStat (SPSS, Inc.) was used for all analyses.

\section{Results}

Between October 1991 and August 2001, 105 children (40 girls, 65 boys) with spastic CP underwent SDR. Average age at surgery was 5 years (range $3-10.5$ years). Of the 105 patients, 102 had formal preoperative evaluations, and 97, 62, 57, and 14 patients received formal post- 
operative assessments at $1,5,10$, and 15 years, respectively. Thus, these patients were followed through their adolescent years and into early adulthood. Sixty-five children were diagnosed with spastic diplegia, 5 with spastic triplegia, and 11 with spastic quadriplegia. The GMFCS classification was not available at the start of the study, and therefore not all children were classified as such preoperatively. Of the 52 children who were classified by GMFCS group preoperatively, 11 were in GMFCS Group I, 22 in Group II, 14 in Group III, and 5 in Group IV. No GMFCS Group V patients were treated using SDR.

\section{Gross Motor Function Measure}

For muscle tone scores (Fig. 1), hip adductor, ankle plantar flexor, and hamstring testing all showed significantly decreased tone (that is, decreased spasticity) throughout the length of follow-up ( $\mathrm{p}<0.001)$ compared with preoperative values. For GMFM assessments relative to preoperative values, GMFM-total, Dimension D, and Dimension E scores all significantly improved at all time points throughout follow-up (Fig. 2A). Gross Motor Function Measure Dimensions A, B, and C are less representative of lowerextremity function but are indicators of overall motor function; Dimensions A, B, and C also significantly improved at all time points throughout follow-up (Fig. 2B).

The GMFM data were also analyzed by stratifying GMFM total scores according to GMFCS groups (Fig. 2C). The GMFCS Groups I, II, and III displayed statistically significant increases through 5- and 10-year follow-up compared with preoperative values. For GMFCS Group IV, there was no significant change in GMFM total scores from preoperative values at any time point. When stratified in this manner there were too few patients in the 15-year follow-up subgroups to perform sound statistical analysis, but the mean values are displayed in Fig. $2 \mathrm{C}$ as points of reference. Preoperative and postoperative GMFCS designations were available for 52 patients. Af-

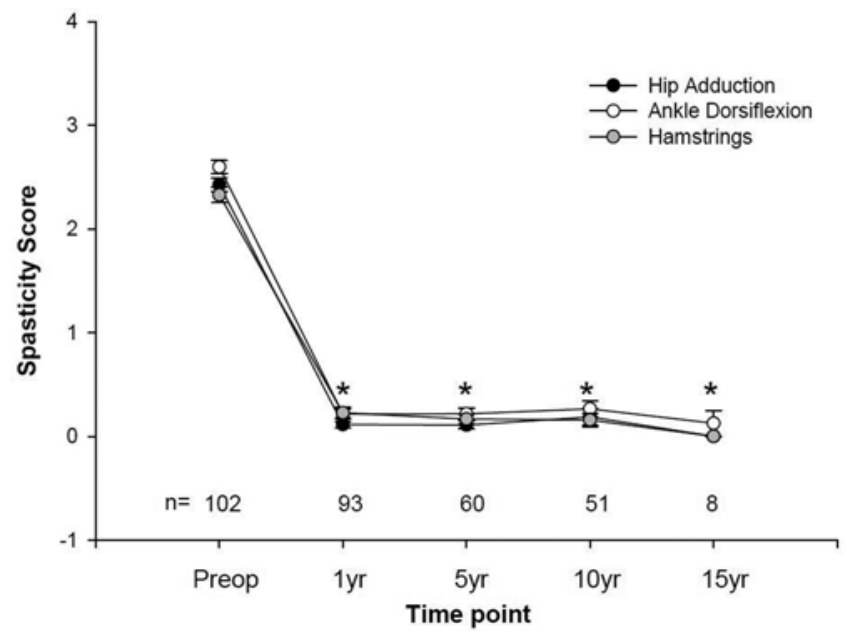

FIG. 1. Line graph of long-term outcomes after SDR for spasticity of hip adductors, hamstrings, and ankle plantar flexors as measured by the modified Ashworth score comparing preoperative values to 1-, 5-, $10-$, and 15-year follow-up values. The number of observations $(n=)$ is displayed along the bottom of the graph. ${ }^{*} p<0.05$ for means at each time point compared with preoperative means. ter SDR, $48(92.3 \%)$ of 52 patients improved to a higher group or remained in the same GMFCS group despite longitudinal growth (data not shown).

\section{Pediatric Evaluation of Disability Inventory}

Compared with preoperative values, PEDI self-care and mobility scaled scores significantly improved throughout the length of follow-up (Fig. 3A). When both PEDI mobility scores and self-care scores were stratified by GMFCS groups (Fig. 3B and C), we found significant improvements through 5- and 10-year follow-up for all groups. Again, when stratified by GMFCS subgroups at 15-year follow-up, too few patients were present in each subgroup to perform statistical analysis for this time point.

\section{Group-Based Trajectory Modeling}

For GBTM, data were analyzed on all 105 children who underwent SDR, and included data collected preoperatively and $1,2,3,5$, and 10 years postoperatively. However, 23 patients with either no GMFM measure or only a single GMFM measure were removed from the analysis. The retained group of 82 patients included mostly children from 3 to 5 years of age, with only 8 older children. While $80(98 \%)$ of the 82 children had GMFM scores recorded at their presurgical visit, the proportion dropped over time to $84 \%$ at 1 year, $70 \%$ at 2 years, $67 \%$ at 3 years, $57 \%$ at 5 years, $50 \%$ at 10 years, and only $13 \%$ at 15 years. The optimal number of trajectory groups identified through GBTM was 4. Mean posterior probabilities of group assignment were high, ranging from 0.93 to 0.99 , supporting excellent fit of the model. The difference between the theoretical proportion in each trajectory group (based on the sum of all posterior probabilities for that group) and calculated group size (based on the group to which an individual was assigned), an indication of classification error, ranged from only $0.06 \%$ to $1.8 \%$ across the groups.

The 4 trajectory groups found, which were based in large part on presurgical GMFM scores, are shown in Fig. 4. Each group showed a fairly linear increase to 3 years, with 3 of the groups continuing to show further increases to 5 years. The 3 trajectories of patients with moderate to high preoperative GMFM scores $(>60)$ maintained their gains at 10 years, but the poorest performing group did not. Sixteen patients $(19.5 \%)$ were in this "poorest" responding group, which began with an average preoperative GMFM score of 43 , reached 59 by 5 years postsurgery, but dropped back to 45 by 10 years. Nineteen $(23.8 \%)$ in a "low-moderate" responding group increased from a preoperative GMFM score of 62 to 75 by Year 5, and remained at 72 at 10 years. Twenty-two children $(27.5 \%)$ in a "high-moderate" responding group started with an average preoperative GMFM score of 68 , rose 20 points over 5 years, and remained at a score of 87 at Year 10. The other 23 children $(28.8 \%)$ in the "best" responding group started with a preoperative GMFM score of 84 , reached a score of 94 by Year 3, and retained their gains to Year 10.

\section{Predictive Index for Long-Term Ambulation After} Rhizotomy

Group assignment was associated with distribution of 


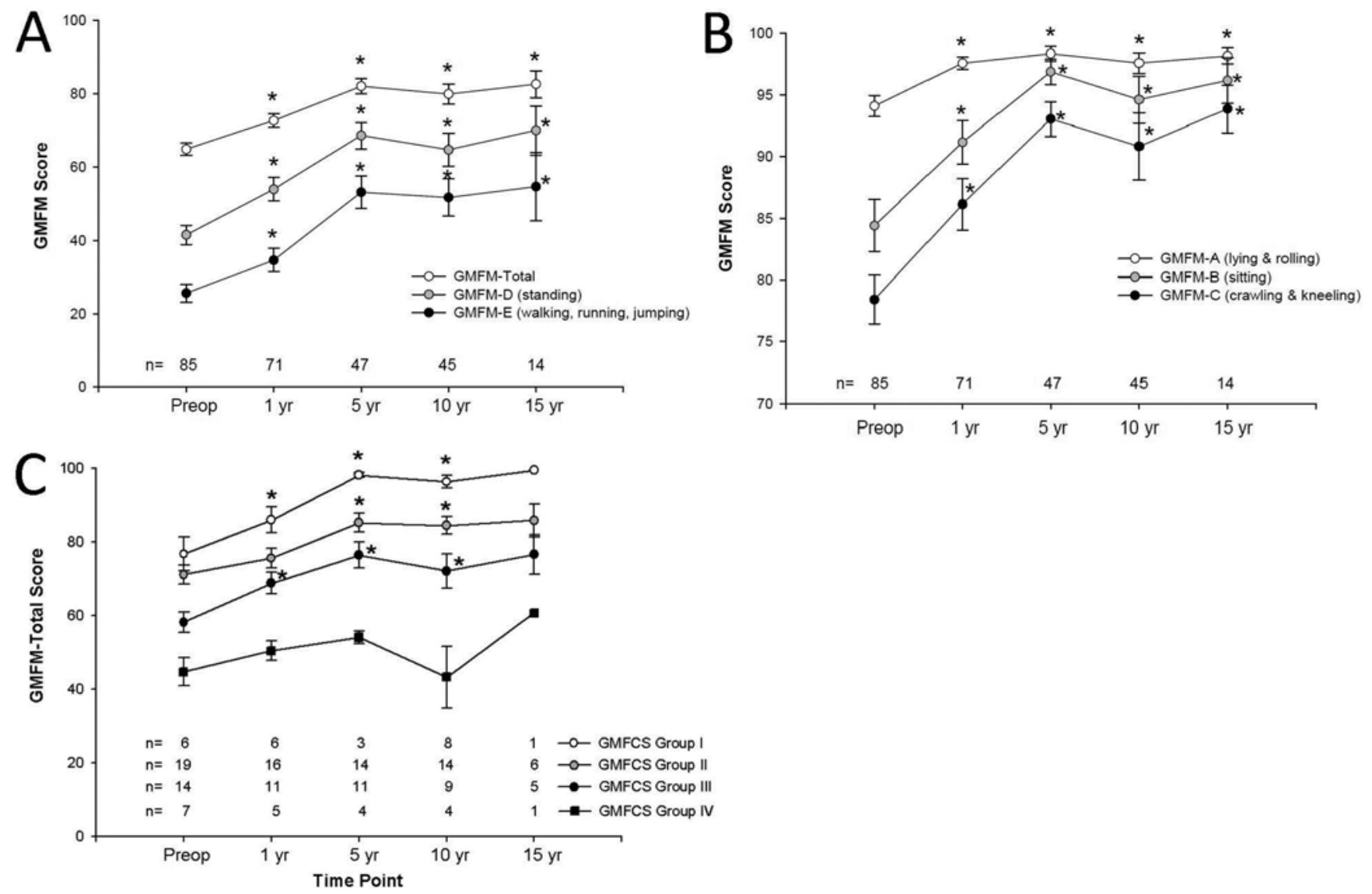

FIG. 2. Line graphs of long-term outcomes after SDR for GMFM comparing preoperative values to 1-, 5-, 10-, and 15-year follow-up values. A: Total GMFM scores, as well as GMFM Dimension D (standing) and Dimension E (walking, running, jumping), which are directly related to lower-limb function. B: GMFM Dimensions A (lying and rolling), B (sitting), and C (crawling and kneeling), which involve upper-limb function as well. C: Total GMFM scores stratified according to CP severity by GMFCS groups. The number of observations in each group $(n=)$ is displayed along the bottom of each graph. * $p<0.05$ for means at each time point compared with preoperative means.

spasticity (diplegia, triplegia, or quadriplegia) and degree of hip adductor spasticity (Ashworth scale 3 vs $<3$ ), but not with age, sex, degree of ankle plantar flexion spasticity, or degree of hamstring spasticity. In the "poorest" performing group, $69 \%$ of patients had spastic quadriplegia. In the "best" performing group, $91 \%$ had hip adductor spasticity of less than 3 on the Ashworth scale, compared with $45 \%, 37 \%$, and $18 \%$ in the "high-moderate," "lowmoderate," and "poorest" responding groups, respectively $(p<0.0001$, degrees of freedom $=3$, chi-square test; and test of trend $p<0.0001)$. We used the results of the associations found with GBTM analysis to create a long-term predictive index for these outcomes, the PILAR (Table 1). The index has 4 components: 1) preoperative GMFCS group assignment, 2) preoperative total GMFM score ( $\leq$ 60 vs $>60$ ), 3) distribution of spasticity (diplegia or triplegia/quadriplegia), and 4) degree of hip adductor spasticity (Ashworth scale 3 vs $<3$ ). Based on preoperative GMFCS group assignments, patients are given from 4 to 0 points in descending order in relation to the GMFCS score. As depicted in Table 1, a GMFCS Group I patient is given 4 points, a GMFCS Group II patient gets 3 points, while a GMFCS Group V patient is assigned 0 points. Each of the other 3 components of the scoring system are "yes" or "no" answers, and therefore are scored 1 or 0 , for the good and bad predictors, respectively, depending on whether the patient has the characteristic or not. For example, if patients have a hip adductor tone score of 3 (a poor predictive factor) they receive 0 points, while if they have any tone score less than 3 (a good predictive factor) they receive 1 point. All 4 scores are added to get a final score. If this final score is greater than or equal to 4 the patient has a very good chance of a long-term beneficial effect on ambulation after SDR. On the other hand, if the score is less than 4 the child has little chance of a good long-term ambulatory outcome with SDR. For ADLs other than ambulation, our PEDI results suggest that even patients with a PILAR score of less than 4 exhibit long-term nonambulatory functional gains, but our index is not designed to address these additional important outcomes. Of course, the index will need to be prospectively validated to confirm if these predictive scores are accurate. However, we already believe that the PILAR is helpful in discussing postoperative long-term expectations with families, both in the ambulatory and nonambulatory domains.

\section{Adjunctive Procedures}

Full records, including details of orthopedic proce- 

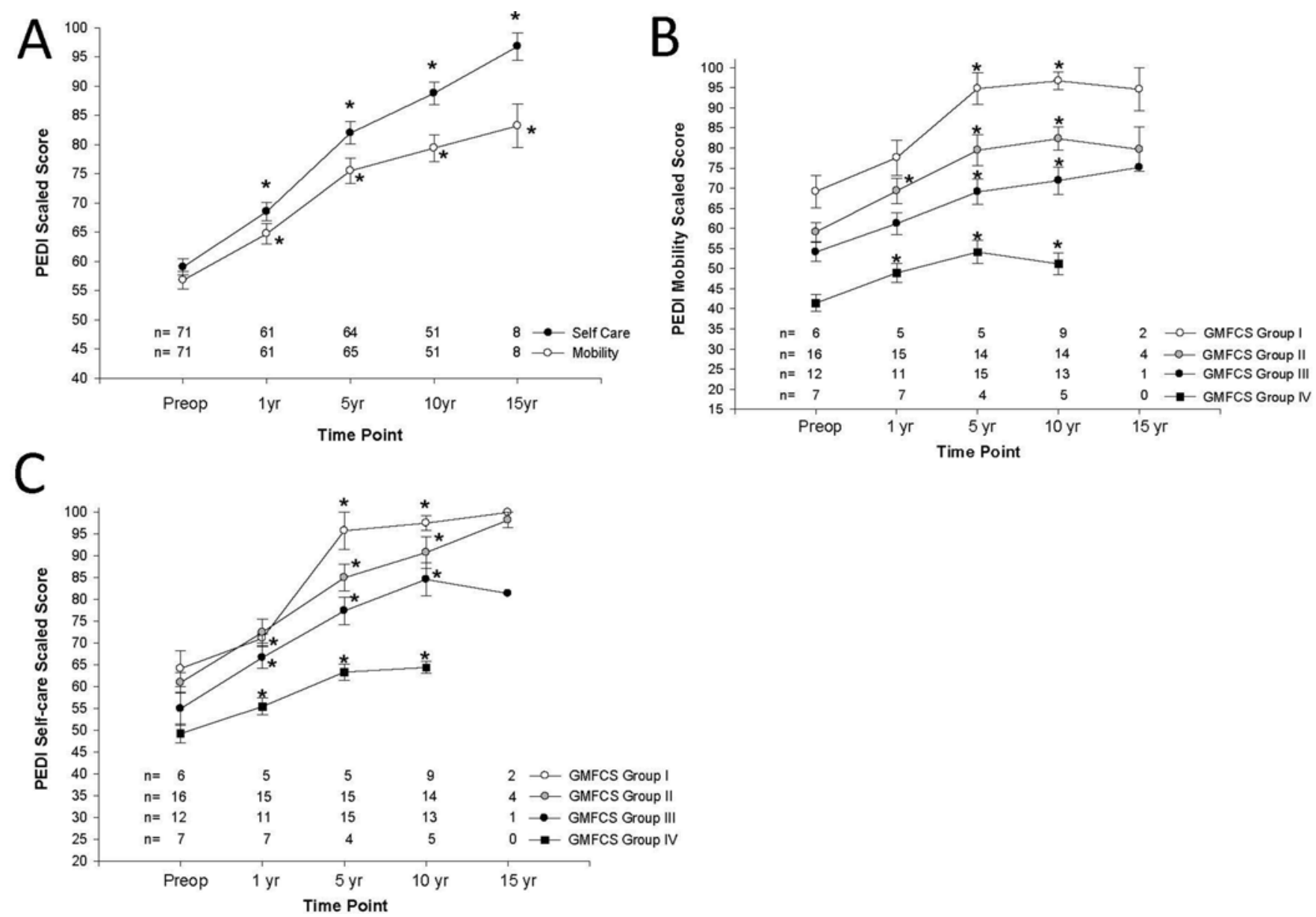

FIG. 3. Line graphs showing long-term outcomes after SDR according to PEDI scores, comparing preoperative values to 1-, 5-, 10-, and 15-year follow-up values. A: Scaled scores for PEDI self-care and mobility for all patients. B: Scaled scores for PEDI mobility stratified according to CP severity by GMFCS groups. C: Scaled scores for PEDI self-care stratified according to CP severity by GMFCS groups. The number of observations in each group $(n=)$ is displayed along the bottom of each graph. ${ }^{*} p<0.05$ for means at each time point compared with preoperative means.

dures and Botox injections, were available and analyzed for 88 of the 105 patients. Of these 88 patients, 52 (59.1\%) had SDR alone, while 11 (12.5\%) had only Botox injections in addition to SDR, leaving 25 patients (28.4\%) needing lower-extremity orthopedic surgery, such as tendon releases and osteotomies, after SDR (Table 2). Importantly, completing the effect of SDR with supplemental tendon release was needed in only 17 patients (19.3\%).

\section{Discussion}

In assessing children with spastic CP long-term, a number of adverse effects related to growth, particularly during the adolescent growth spurt, can lead to a progressive deterioration of the early good release of spasticity. ${ }^{4,10}$ Outcome studies must consider the natural history of motor development in growing children with CP. In the population-based Ontario Motor Growth Study and the Adolescent Study of Quality of Life, Mobility, and Exercise, Rosenbaum et al..$^{18}$ and Hanna et al..$^{10}$ developed predictive models of GMFM scores stratified by GMFCS groupings. From these models it was shown that untreated children with spastic CP on average reach $90 \%$ of their motor function at approximately 5 years of age, then plateau in further motor development at about 7 years of age, and children in GMFCS Groups III, IV, and V suffer a decline through adolescence.

We have presented objective functional outcomes through adolescence on a large number of patients in whom we could stratify GMFM data by GMFCS groups. Our results show that spasticity is eliminated after SDR and that the effect on spasticity and gross motor function is durable through adolescence. Unfortunately, we cannot directly compare our data to published natural history curve ${ }^{10}$ in a quantitative manner due to the fact that these more recent natural history curves use a newer version of the GMFM (GMFM-66). However, we can make some general descriptive observations in relation to these natural history studies. First, our SDR-treated children did not plateau in terms of GMFM scores as early as noted in the natural history curves. Both in our GMFM graphs stratified by GMFCS, and in our GBTM graph, it can be observed that GMFM scores in our SDR-treated children do not plateau until 5 years after the surgery when they are, on average, 10 years of age. Second, not only do our SDR-treated patients in GMFCS Groups I and II main- 
R. W. R. Dudley et al.

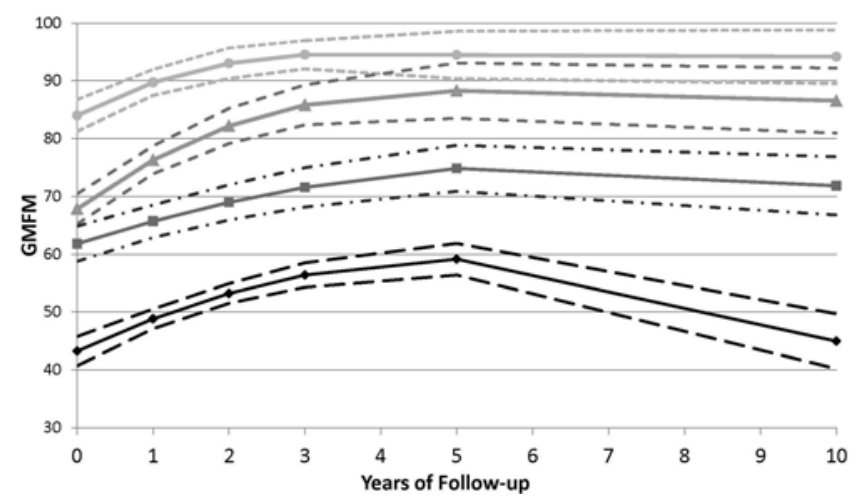

FIG. 4. Line graph of GBTM reveals 4 patient groups with statistically significant differences in GMFM outcomes following SDR. Based on these trajectories, from top to bottom, these groups were labeled the "best" responding group, the "high-moderate" responding group, the "low-moderate" responding group, and the "poorest" responding group, respectively. Mean posterior probabilities of group assignment were high, ranging from 0.93 to 0.99 , supporting excellent fit of the model.

tain their GMFM scores through adolescence, but so do our GMFCS Group III patients, which suggests that SDR may be protective against adolescent declines observed in this group in the natural history curves.

Overall, our results suggest that if patients in GMFCS Groups I, II, and III undergo SDR at approximately 5 years of age, this will allow for continued functional improvements in terms of ambulation in childhood and protection

\section{TABLE 1: Predictive Index for Long-term Ambulation after Rhizotomy}

\begin{tabular}{lc}
\hline \multicolumn{1}{c}{ Factor } & Points \\
\hline preop GMFCS group & 4 \\
II & 3 \\
III & 2 \\
V & 1 \\
score & 0 \\
preop GMFM score & \\
$>60$ & \\
$\leq 60$ & 1 \\
score & 0 \\
spasticity distribution & \\
spastic diplegia & \\
spastic triplegia/quadriplegia & 1 \\
score & 0 \\
hip adductor tone (Ashworth Scale) & \\
$<3$ & \\
3 & \\
score & 1 \\
total score* &
\end{tabular}

* Total scores $\geq 4$ = good chance of long-term functional improvement in ambulation after SDR; scores $<4=$ poor chance of long-term functional improvement in ambulation after SDR.
TABLE 2: Adjunctive procedures following SDR

\begin{tabular}{lc}
\hline \multicolumn{1}{c}{ Procedure } & No. of Patients (\%) \\
\hline SDR & $88(100)$ \\
SDR alone & $52(59.1)$ \\
SDR \& botox alone & $11(12.5)$ \\
adjunctive lower limb orthopedic surgery & $25(28.4)$ \\
$\quad$ bone (osteotomies) & $14(15.9)$ \\
soft tissue (tendon release) & $17(19.3)$ \\
$\quad$ both bone \& soft tissue & $6(6.8)$ \\
bracing or surgery for spinal deformities & $1(1.1)$ \\
\hline
\end{tabular}

against declines through adolescence. In a subsample of 52 patients for whom preoperative and postoperative GMFCS designations were available, we found that $92.3 \%$ either improved or maintained their GMFCS designation throughout long-term follow-up. This finding is noted despite the fact that, inherent to the GMFCS classification system, a patient who uses a walker before age 4 is classified as a GMFCS Group II patient, but automatically becomes a GMFCS Group III patient when he or she surpasses the age of 4 if still using the walker. ${ }^{17}$ We cannot overstate the importance of the intensive rehabilitation that is performed early after SDR. However, it is unlikely that physiotherapy alone can explain the long-term benefits observed here, as few patients, if any, continue such efforts at 5, 10, and 15 years after surgery.

To verify our findings using a statistical method that could address missing data, we employed GBTM. ${ }^{15}$ Not only did this analysis confirm the long-term efficacy of SDR on GMFM scores, but it divided our population into 4 subgroups that each had a different course of GMFM outcome over time. Interestingly, these 4 "trajectories" very closely resembled the patterns of outcomes observed when the population was stratified by GMFCS group, despite the fact that GMFCS was not included as a variable in the model. Not surprisingly, the division of the population into these outcome subgroups was associated with markers of preoperative spasticity severity. Greater distribution of spasticity (spastic quadriplegia) and severe proximal lower-extremity spasticity (maximum adductor spasticity) were associated with the "poorest" performing group, which did not show durable functional benefits through adolescence.

Sustainable improvements observed here in GMFM Dimensions A, B, and C (lying and rolling, sitting, and crawling and kneeling, respectively) suggests that functional gains are not restricted purely to lower-limb functions. The multidimensional benefits of SDR are emphasized in our PEDI results, which revealed significant gains for both self-care and mobility that persisted through adolescence and into early adulthood. When stratified by GMFCS, Groups I-IV showed durable PEDI improvements through adolescence. These are very important findings, because it suggests that after SDR, these children are able to develop significant skills that allow them to adequately perform their ADLs and gain a reasonable amount of independence as they develop into young adults. In addition, when health care professionals 
and families are considering SDR, another option that is often discussed is intrathecal baclofen pumps, which have the perceived advantage of being more beneficial to the upper extremities than a surgical procedure that manipulates only lumbar and sacral nerve roots. ${ }^{3}$ However, our data suggest there are significant and durable benefits to upper extremity-related functions as well, after SDR.

Children with spastic CP often require orthopedic surgical procedures to correct spasticity-related lowerextremity deformities. ${ }^{5,22}$ In a prospective study, Watt et al. ${ }^{22}$ showed that $61 \%$ of untreated children (no SDR) with spastic CP undergo orthopedic surgical procedures before the age of 8 . In our long-term follow-up in this study, $28.4 \%$ of patients who underwent SDR required adjunctive orthopedic surgical procedures, while more than $70 \%$ of children received either SDR alone or with only Botox injections as an adjunct procedure. In addition, when we excluded derotation osteotomies and other bone procedures for deformities that occur due to spasticity in the early years of life (prior to SDR), we found that only 17 patients $(19.3 \%)$ required tendon release procedures to enhance the effects of SDR.

The main limitation of this study is the lack of a control group, which could potentially introduce bias. However, 2 of 3 previous randomized control trials and a metaanalysis of those trials have shown the short-term benefits of SDR and physiotherapy compared with physiotherapy alone. Of course, it is always possible that patients who undergo SDR and their families are more motivated, or have better support, or better access to various adjunct therapies, which could contribute to the success of SDR. Although our data were analyzed retrospectively, they were collected prospectively by experienced therapists, most of whom are not coauthors on this study. Finally, all cases operated on and all data collected were included in our analysis. The decrease in the number of patients presented at each time point was, for the most part, not because of patient attrition, but rather that patients had not yet reached the 10-year, and particularly the 15-year, follow-up. Nevertheless, the relatively large number of patients included, and the well-validated assessment tools used, provide the most rigorous means of testing our hypothesis within the confines of an uncontrolled case series, and provide a good level of confidence in our results.

\section{Conclusions}

Our results indicate that SDR is an efficacious procedure that allows for long-lasting improvements in spasticity, gross motor function, and performance of ADLs. In addition, according to our confirmatory GBTM results, SDR will have a durable protective effect on ambulation, particularly for spastic diplegic children with preoperative GMFM scores greater than 60 and hip spasticity scores less than 3 , which corresponds to the group of children with GMFCS Group I, II, and III preoperative levels. These important predictive factors are emphasized in the PILAR (Table 1). Together, these results suggest that if we operate on such well-selected children before their gross motor development plateaus we may be able to protect them from adolescent declines and allow them to improve and maintain function into early adulthood. Therefore, higher functioning children will remain higher functioning adolescents and young adults.

\section{Disclosure}

The authors report no conflict of interest concerning the materials or methods used in this study or the findings specified in this paper.

Author contributions to the study and manuscript preparation include the following. Conception and design: Farmer. Acquisition of data: Farmer, Dudley, Parolin, Saluja, Yap, Montpetit, Ruck. Analysis and interpretation of data: Farmer, Dudley, Parolin, Saluja, Yap, Ruck, Poulin. Drafting the article: Farmer, Dudley, Parolin, Gagnon. Critically revising the article: all authors. Reviewed submitted version of manuscript: all authors. Approved the final version of the manuscript on behalf of all authors: Farmer. Statistical analysis: Dudley, Parolin, Gagnon, Saluja. Study supervision: Farmer.

\section{References}

1. Abbott R, Johann-Murphy M, Shiminski-Maher T, Quartermain D, Forem SL, Gold JT, et al: Selective dorsal rhizotomy: outcome and complications in treating spastic cerebral palsy. Neurosurgery 33:851-857, 1993

2. Aisen ML, Kerkovich D, Mast J, Mulroy S, Wren TA, Kay RM, et al: Cerebral palsy: clinical care and neurological rehabilitation. Lancet Neurol 10:844-852, 2011

3. Albright AL, Barry MJ, Fasick MP, Janosky J: Effects of continuous intrathecal baclofen infusion and selective posterior rhizotomy on upper extremity spasticity. Pediatr Neurosurg 23:82-85, 1995

4. Bottos M, Feliciangeli A, Sciuto L, Gericke C, Vianello A: Functional status of adults with cerebral palsy and implications for treatment of children. Dev Med Child Neurol 43: 516-528, 2001

5. Chicoine MR, Park TS, Kaufman BA: Selective dorsal rhizotomy and rates of orthopedic surgery in children with spastic cerebral palsy. J Neurosurg 86:34-39, 1997

6. Cosgrove AP, Graham HK: Botulinum toxin A prevents the development of contractures in the hereditary spastic mouse. Dev Med Child Neurol 36:379-385, 1994

7. Farmer JP, Sabbagh AJ: Selective dorsal rhizotomies in the treatment of spasticity related to cerebral palsy. Childs Nerv Syst 23:991-1002, 2007

8. Golan JD, Hall JA, O'Gorman G, Poulin C, Benaroch TE, Cantin MA, et al: Spinal deformities following selective dorsal rhizotomy. J Neurosurg 106 (6 Suppl):441-449, 2007

9. Haley SM, Raczek AE, Coster WJ, Dumas HM, FragalaPinkham MA: Assessing mobility in children using a computer adaptive testing version of the pediatric evaluation of disability inventory. Arch Phys Med Rehabil 86:932-939, 2005

10. Hanna SE, Rosenbaum PL, Bartlett DJ, Palisano RJ, Walter $\mathrm{SD}$, Avery L, et al: Stability and decline in gross motor function among children and youth with cerebral palsy aged 2 to 21 years. Dev Med Child Neurol 51:295-302, 2009

11. Jones BL, Nagin DS: Advances in group-based trajectory modeling and an SAS Procedure for estimating them. Sociol Methods Res 35:542-571, 2007

12. McLaughlin J, Bjornson K, Temkin N, Steinbok P, Wright V, Reiner A, et al: Selective dorsal rhizotomy: meta-analysis of three randomized controlled trials. Dev Med Child Neurol 44:17-25, 2002

13. McLaughlin JF, Bjornson KF, Astley SJ, Graubert C, Hays RM, Roberts TS, et al: Selective dorsal rhizotomy: efficacy and safety in an investigator-masked randomized clinical trial. Dev Med Child Neurol 40:220-232, 1998

14. Mittal S, Farmer JP, Al-Atassi B, Gibis J, Kennedy E, Galli C, et al: Long-term functional outcome after selective posterior rhizotomy. J Neurosurg 97:315-325, 2002 


\section{R. W. R. Dudley et al.}

15. Nagin DS, Odgers CL: Group-based trajectory modeling in clinical research. Annu Rev Clin Psychol 6:109-138, 2010

16. Nordmark E, Josenby AL, Lagergren J, Andersson G, Strömblad LG, Westbom L: Long-term outcomes five years after selective dorsal rhizotomy. BMC Pediatr 8:54, 2008

17. Palisano R, Rosenbaum P, Walter S, Russell D, Wood E, Galuppi B: Development and reliability of a system to classify gross motor function in children with cerebral palsy. Dev Med Child Neurol 39:214-223, 1997

18. Rosenbaum PL, Walter SD, Hanna SE, Palisano RJ, Russell DJ, Raina P, et al: Prognosis for gross motor function in cerebral palsy: creation of motor development curves. JAMA 288: 1357-1363, 2002

19. Russell DJ, Rosenbaum PL, Cadman DT, Gowland C, Hardy $\mathrm{S}$, Jarvis S: The gross motor function measure: a means to evaluate the effects of physical therapy. Dev Med Child Neurol 31:341-352, 1989

20. Steinbok P, Reiner AM, Beauchamp R, Armstrong RW, Cochrane DD, Kestle J: A randomized clinical trial to compare selective posterior rhizotomy plus physiotherapy with physiotherapy alone in children with spastic diplegic cerebral palsy. Dev Med Child Neurol 39:178-184, 1997

21. Ubhi T, Bhakta BB, Ives HL, Allgar V, Roussounis SH: Randomised double blind placebo controlled trial of the effect of botulinum toxin on walking in cerebral palsy. Arch Dis Child 83:481-487, 2000

22. Watt JM, Robertson CM, Grace MG: Early prognosis for ambulation of neonatal intensive care survivors with cerebral palsy. Dev Med Child Neurol 31:766-773, 1989

23. Wright FV, Sheil EM, Drake JM, Wedge JH, Naumann S: Evaluation of selective dorsal rhizotomy for the reduction of spasticity in cerebral palsy: a randomized controlled tria. Dev Med Child Neurol 40:239-247, 1998

Manuscript submitted November 15, 2012.

Accepted April 11, 2013.

Portions of this paper were presented at the 47th Annual Congress of the Canadian Neurological Sciences Federation on June 7, 2012, in Ottawa, Ontario, Canada, and at the 2012 Congress of Neurological Sciences Annual Meeting on October 9, 2012, in Chicago, Illinois.

Please include this information when citing this paper: published online May 28, 2013; DOI: 10.3171/2013.4.PEDS12539.

Address correspondence to: Jean-Pierre Farmer, M.D., C.M., F.R.C.S.(C), Division of Neurosurgery, Department of Pediatric Surgery, Montreal Children's Hospital, McGill University, 2300 Tupper Street, Room C-1139, Montreal, Quebec, Canada H3H 1P3. email: jeanpierre.farmer@muhc.mcgill.ca. 